\title{
Immersive Edition of Multisensory 360 Videos
}

\author{
Hugo Coelho $^{1}$, Miguel Melo ${ }^{2(凶)}$, Luís Barbosa ${ }^{1,2}$, José Martins ${ }^{1,2}$, \\ Mário Sérgio $^{1}$, and Maximino Bessa ${ }^{1,2}$ \\ 1 Universidade de Trás-os-Montes e Alto Douro, Vila Real, Portugal \\ 2 INESC TEC, Porto, Portugal \\ mcmelo@inesctec.pt
}

\begin{abstract}
The current technologic proliferation has originated new paradigms concerning the production and consumption of multimedia content. This paper proposes a multisensory 360 video editor that allows producers to edit such contents with high levels of customization. This authoring tool allows the edition and visualization of 360 video with the novelty of allowing to complement the 360 video with multiple stimuli such as audio, haptics, and olfactory. In addition to this multisensory feature, the authoring tool allows customizing individually each of the stimuli to provide an optimal multisensory user experience.

A usability evaluation has revealed the pertinence of the editor, where it was verified an effectiveness rate of $100 \%$, only one help request out of 10 participants, and positive efficiency. Satisfaction-wise, results equally revealed high level of satisfaction as the average score was 8.3 out of 10 .
\end{abstract}

Keywords: Virtual reality · Multisensory 360 video

Interactive edition

\section{Introduction}

The evolution of technology has led to the appearance of new paradigms regarding multimedia content. An example of this is the growing interest in 360 videos, motivated in part by the fact that display devices such as head-mounted displays (HMDs), which initially had technical limitations and high prices, have evolved to a point that they are capable of delivering good user experience as well as they became affordable to the consumer. In fact, there are market research that show the growth of this type of equipment in the consumer market: it is estimated that by 2020 this market share will reach around $€ 13.5$ billion [11].

Unlike traditional video that limits the viewer's view to the viewing angle of the camera, 360 video gives the viewer the opportunity to look around freely as if he/she was in the place where the depicted action occurs [1]. Such feature affects user experience as it can have an emotional effect on users, namely at the level of immersion and involvement [16]. In most cases, the 360 video is complemented with audio that can be stereo or even spatialized (the sound is processed taking into account users' head movement).

(C) Springer International Publishing AG, part of Springer Nature 2018

Á. Rocha et al. (Eds.): WorldCIST'18 2018, AISC 746, pp. 309-318, 2018.

https://doi.org/10.1007/978-3-319-77712-2_30 
It is important to say that 360 video is not limited to the use of omnidirectional video complemented with an audio stimulus. In fact, there is already works that follow a multisensory approach, complementing these two stimuli with more stimuli such as, for example, haptic and/or olfactory stimuli. An illustrative example of multisensory video 360 is the work developed by Ramalho and Chambel [14] that considered haptic stimuli, namely, wind simulation. This work evaluated the sense of presence and experienced realism and results have revealed that such multisensory approach had an impact on these variables. The study obtained also positive feedback from the participants towards this multisensory approach. Another approach to multisensory 360 video was proposed by [9] that delivers haptic feedback that, after a proper customization, can be consistent with the content that is being displayed. The hardware consists of a chair (where the user should be seated) in which a matrix of eight vibrating points is mounted and the haptic feedback is delivered in the form of vibrations.

Although there are already some proposals for multisensory 360 video content, there is no established authoring tool that allows the creation of multisensory experiences in an intuitive way. It should be noted that such tools represent challenges such as ensuring the timing of the different stimulus to be delivered in a way that ensures consistency between the different stimuli. This will benefit the user experience and thus achieve high levels of immersion and credibility [7].

One of the few works that allow authoring multisensory experiences based on 360 videos was proposed by Freitas et al. [6]. Besides 360 video and audio, this work considers haptic feedback (wind simulation) and olfactory feedback. The authoring tool allows the customization of the experience by defining which stimulus is going to be delivered and their duration. However, this work presented some limitations: limited tracking of horizontal movements $\left(180^{\circ}\right)$ that does not allow the full 360 view of the 360 videos. Another limitation is related to the olfactory stimulation: it is not possible to control the intensity of the smell. Despite the support for haptic feedback, the authors stated that did not test it due to hardware constraints.

Given the growing popularity of this new type of content, and considering that the state-of-the-art is scarce, it becomes important to develop mechanisms that allow the authoring of multisensory 360 video experiences in an expeditious and intuitive manner. Taking this as motivation, this paper proposes an interactive authoring tool for multisensory 360 videos. The proposed authoring tool aims to allow the customization of 360 videos by allowing to add: sound clips in a complementary way to the original 360 audio track; haptic stimuli through wind simulation and force feedback; and olfactory stimuli that can be customized to allow the delivery of up to 2 different smells simultaneously. An added value of the present proposal in relation to the work proposed by Freitas et al. [6] is that it allows to previewing the multisensory experience "in loco" while the experience is being created. The possibility of previewing the experience is important because it allows, in real time, to see the effect that certain stimulus induces and to fine-tune them in order to match the content producer expectations. 
When developing such innovative tools, it is important to ensure its usability. By usability is meant the ease of use that can be observed through the effectiveness, efficiency, and satisfaction of the users about a particular application/system. Thus, a usability assessment consists in the evaluation of these same parameters that, according to [8], can be defined to define as follows:

- Effectiveness: the effectiveness and precision with which the user can achieve the proposed objectives;

- Efficiency: resources spent to successfully reach the proposed objectives;

- Satisfaction: comfort and acceptance of system operation by users.

The most common practices for usability evaluation are direct observation, the error rate and the number of requests for help [5]. In addition, there are also several usability questionnaires for the purpose of measuring the efficiency and effectiveness of users such as the System Usability Scale (SUS) [2], Software Usability Measurement Inventory (SUMI) [10] or the Questionnaire for User Interaction Satisfaction (QUIS) [12].

Therefore, in addition to the proposal of the multisensory 360 video authoring tool, this paper also presents a pilot study focused at the usability of the system to identify the ease of use and the feasibility of the proposed tool. To develop efforts to optimize the proposed authoring tool in order to enhance its functionality, debriefing interviews were also held to collect the users' feedback.

\section{Immersive Multisensory 360 Video Editor}

For the development of the interactive multisensory 360 video authoring tool, requirement analysis was made to identify the pertinent core features and to proceed to a planned development based on a reflection-action methodology.

\subsection{Requirement Analysis}

To define an informed requirement analysis, one considered the work of Freitas et al. [6]. Having this into account, one defined a set of functional and nonfunctional requirements to guide the development of a multisensory 360 authoring tool designed to enable the user to create experiences based on 360 videos and that can be complemented with various stimuli: sounds, haptic feedback and smell. The authoring tool is also designed to allow a real-time preview of the experiments as well as their visualization once created.

Thus, the editor should allow the user to create a new project or edit a project. After that, the editor should allow the user to import a 360 video as the basis of the project. Having this starting point, the user is able to add new stimulus to the project, specifying the start time and end time. For instance, if it is a sound stimulus, the user should be able to import a sound file with the possibility of previewing it. The user can edit or remove any previously added stimulus. The editor should list all the stimuli added to the project as well as 


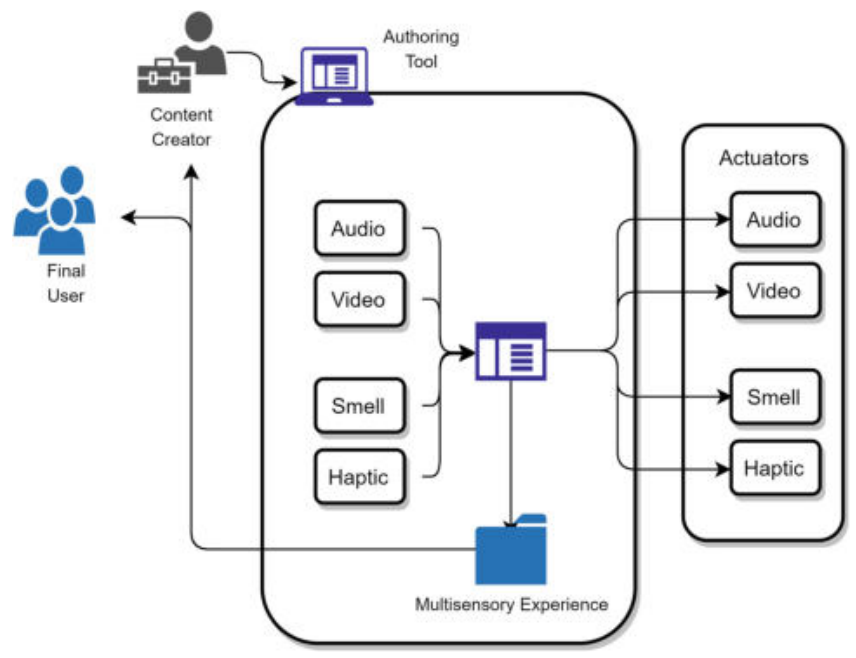

Fig. 1. Multisensory 360 video editor scheme.

the timeline for all the stimuli. At any given time, the user can change the video file and keep all the added stimuli in the project. The editor should do the basic functions of video playback (play, pause, restart, change frame). In addition, the editor should allow the user to save the project to a .xml file and copy all the files needed to a folder with the project name. Everything said above has to be made available through an intuitive and easy to use graphical user interface (GUI). For the proper stimulus delivery, the editor should communicate with the hardware responsible for each stimulus.

The proposed architecture is shown in Fig. 1. First, the Content Creator interacts with the Authoring Tool to add/edit stimuli to the multisensory experience. The Authoring Tool processes all the information through a Multisensory Integrator that ensures the proper handling of all the stimuli that integrate the multisensory experience and ensures that they are properly delivered through the different actuators. The novelty of this system is that the multisensory experience can be pre-viewed "in-loco" by the Content Creator, who can fine-tune it in real-time. Once the Content Creator decides that the Multisensory Experience is ready to be distributed, the Final User can experience it.

\subsection{Prototype Implementation}

The research team adopted Unity3D cross-platform 3D engine for the development of the system as the user-friendly development environment is VR ready that enabled us to develop a robust prototype.

Figure 2 shows GUI of the main screen of the editor. At the center of the top area, it is possible to preview the selected 360 video. On the left side of the video pre-visualization area, there are four buttons: "Open Project", "Add 


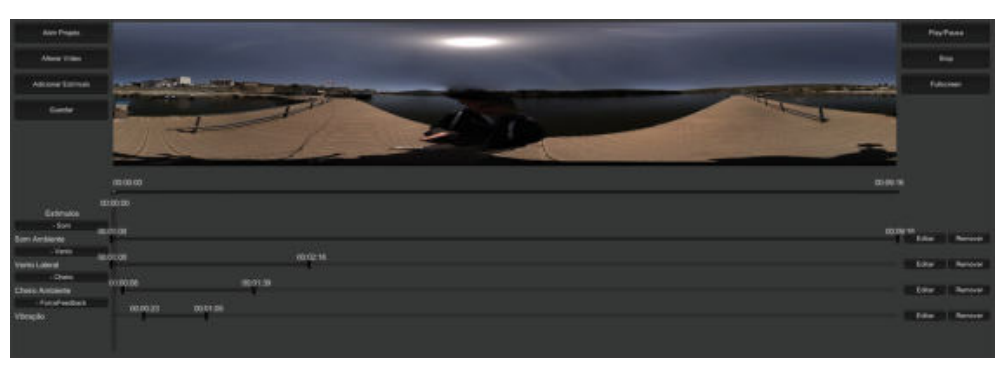

Fig. 2. Multisensory 360 video interactive editor home screen.

Video", "Add Stimulus" and "Save". On the right, there are three additional buttons: "Play/Pause", "Stop" and "Fullscreen". The functionality of each button is self-explained by its name. Below this top area, there are a series of elements illustrated where each one corresponds to example stimuli that compose the multisensory experience that is being depicted and where it is possible to see also a set of controls associated to each of the stimulus (sliders to adjust duration of the stimulus and edit/remove buttons). The different stimulus was included in the prototype as follows:

- Visuals: inclusion of 360 videos in the multisensory experience;

- Audio: sound clips in mono, stereo, and 3D formats;

- Haptics: wind simulation and force feedback;

- Smell: up to 3 different smells to the multisensory experience.

When adding any stimuli, it is asked to configure all the associated parameters. For instance, when adding a haptic stimulus (wind), one can define the name of the stimulus, its intensity, direction, and duration. A preview of the video is also presented in to be used as a reference for the timings.

\section{Evaluation of the Proposed Solution}

The evaluation consisted of an usability study, as described below.

\subsection{Sample}

The evaluation of the multisensory 360 video editor was performed by 10 subjects ( 8 males and 2 females) of the young adult age group (between 18 and 40 years). All participants reported normal or corrected-to-normal vision. None of the participants reported any kind of auditory or olfactory disabilities. 


\subsection{Materials}

Besides the multisensory 360 video editor, the following materials were used:

- Oculus Rift CV 1 [13]: this HMD allows full tracking of head movements, a viewing angle of $110^{\circ}$, and a resolution per eye of $1080 \times 1200$. The sound was delivered using the built-in headphones;

- Buttkicker LFE Kit [3]: this device consists of a transducer that allows the delivery of haptic stimulation through force feedback;

- Sensory Co SmX-4D [15]: a professional solution for aromas' delivery that allows the customized delivery of up to 3 aromas;

- Wind simulator: custom system built by the research team that allows having control over the intensity and duration of the flow of air to be delivered from 4 different points (North, South, East, West);

- Desktop PC: the evaluation took place on a computer consisting of an Intel i7-5820K CPU, three NVIDIA 980 GPUs, 32 GB RAM together with an ASUS VX248H 24" FHD monitor.

To evaluate the usability of the system, the usability questionnaire SUS [2] was used. This questionnaire is composed of 10 items that must be classified on a Likert scale of 1 to 10 where 1 corresponds to "Disagree Totally" and 10 to "I totally agree". For the interpretation of the questionnaires, the authors divide the classification into 5 percentiles, highlighting the following:

- Percentile A: scores above 80.3. It is considered to be a system that users like to use and would recommend to others;

- Percentile C: scores above 68. It is the minimum acceptable limit to consider that the system has a satisfactory usability;

- Percentile F: scores below 51 mean that the system has serious weaknesses in usability that should be corrected as soon as possible.

As defined by the SUS questionnaire's authors [2], the usability indexes were calculated as follows: for each of the odd-numbered questions, one subtracted 1 from the score; for each of the even numbered questions, subtracted their value from 5; the new values which were calculated were summed and multiplied by 2.5. The SUS questionnaire was complemented with a set of metrics that were registered by means of direct observation, namely: number of errors committed, help requests, total time needed for the completion of the proposed procedure, and overall satisfaction of the users over the system's usage.

\subsection{Procedure}

The experiments took place in an experimental room where are the environmental variables were controlled by the research team: no noise, controlled temperature, and ambient lighting of $500 \mathrm{~cd} / \mathrm{m}^{2}$ as recommended by the Illuminating Engineering Society to indoor working environments that demand good visual acuity [4]. When the participant entered the experimental room, he was directed 


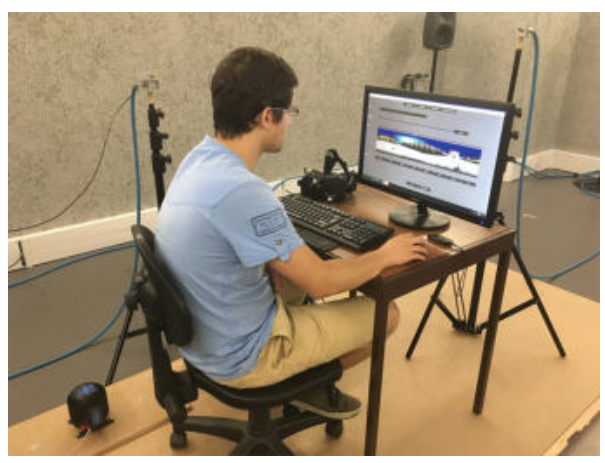

Fig. 3. Participant using the editor (illustrative photo).

to the experimental apparatus: a table in the center of the room, with a monitor, a mouse, a keyboard and the HMD. The various devices for the stimulation of the various senses were also properly setup around the user's place (Fig. 3).

Before starting the experimental protocol, a briefing was given to the participant about the multisensory 360 video editor and its features with the possibility of clarifying any possible doubts about the software. Then, it was given to the participant an experimental protocol with the following tasks:

- Create a new multisensory experience project;

- Add the "ReguaJuntoRio.mp4" 360 video to the multisensory experience;

- Add the "Som_Ambiente.wav" sound clip to the multisensory experience;

- Define the sound clip to play between the 00:00:05 and 00:01:00;

- Add a haptic stimulus (wind) to experience named "Vento" with a level 7 of intensity and to be displayed from 00:00:10 to 00:00:45;

- Add an olfactory stimulus named "Cheiro Floral" located at Capsule 1 starting at 00:00:00 and being delivered until the moment 00:01:00;

- Add a haptic stimulus (force feedback) with the name "Vibracao" and a level 5 intensity starting at 00:00:015 and stopping at 00:01:00;

- Save the multisensory experience on the desktop with the name "AproveiteEsteMomento.massiveFile";

- Open and play the newly-created multisensory experience.

At any point, participants could preview the experience they were creating through HMD. The accomplishment of the protocol was accompanied by a member of the research team who registered, by means of direct observation, the time spent and the number of errors committed. The participant was also informed that at any time, in case of any doubt, he could ask for help to the member of the research team present in the room (who counted the help requests). At the end of the experiment, a small debriefing was made with the participants to gather some feedback on the multisensory 360 video editor to identify possible improvements to be made and new features to be implemented. 


\subsection{Variables}

The independent variable of this study is the multisensory video editor itself. As for the dependent variables, these are: the usability of the system (index provided by the SUS questionnaire application); effectiveness (complete the experiment successfully, number of errors and number of help requests); efficiency (time spent on the implementation of the protocol); and satisfaction.

\section{Results}

The results of the experimental study are reported in the Table 1.

Table 1. Results of the evaluation of the immersive multisensory 360 video editor

\begin{tabular}{l|l|l|l|l|l}
\hline & SUS index & No. of errors & Help requests & Total time $(\mathrm{min})$ & Satisfaction \\
\hline Participant 1 & 71 & 0 & 0 & 8 & 9 \\
\hline Participant 2 & 70 & 0 & 0 & 6 & 7 \\
\hline Participant 3 & 87 & 0 & 0 & 7 & 8 \\
\hline Participant 4 & 92 & 0 & 0 & 8 & 10 \\
\hline Participant 5 & 80 & 0 & 0 & 7 & 8 \\
\hline Participant 6 & 84 & 0 & 0 & 6 & 8 \\
\hline Participant 7 & 75 & 0 & 0 & 8 & 8 \\
\hline Participant 8 & 92 & 0 & 1 & 7 & 10 \\
\hline Participant 9 & 95 & 0 & 0 & 8 & 10 \\
\hline Participant 10 & 70 & 0 & 0 & 6 & 5.3 \\
\hline Average & 81.6 & 0 & 0.1 & 7.1 & 5 \\
\hline
\end{tabular}

Regarding the usability index, it was calculated through the participants' answers to the SUS questionnaire. For all participants, the usability index was always over 68 points (minimum reference value identified by the authors of the SUS questionnaire), the mean obtained being 81.6 points.

As regards the errors made, it should be noted that there was no error made by the participants in the creation of the multisensory video 360 experience. Regarding requests for help, of the 10 participants there was only one participant who requested help from the researcher in the experimental room. The average duration of the creation of the proposed experiment was about $7 \mathrm{~min}$. The average degree of satisfaction was 8.3 points on a scale of 1 to 10 where 1 corresponds to "Not Satisfied" and 10 to "Completely Satisfied".

\section{Discussion}

The evaluation of the multisensory 360 video editor was focused on its usability. Results revealed that the average usability index reported was of 81.6 points, 
which corresponds to a Percentile A classification. Such result demonstrates the feasibility of the proposed system and that, according to the original evaluation, participants considered the authoring tool proposed a pleasant system to use and would possibly recommend its use. A detailed analysis of the results allows to verify that the reported usability indexes were always higher than 68 points, thus demonstrating that the system has no significant usability problems according to the original evaluation grid of the usability questionnaire.

Regarding efficacy, there was $100 \%$ efficacy since all participants were able to correctly perform the experimental protocol. This is important as it demonstrates that the proposed solution allows the creation of content without problems even for users who are using it for the first time. Regarding the number of errors, the results were also solid: 0 errors made. As for help requests, there was only 1 request: one of the participants asked for clarification on how to adjust the duration of a given stimulus. We attribute these positive results to the development methodology adopted: an action-reflection-action approach allowed to have a number of iterations with the input of specialists from the content production and software development areas to improve the proposed solution.

As for the "Total Time" efficiency variable, it was defined that a positive value would be a duration between 4 min (estimated time that an experienced user with the editor takes to do the protocol) and $8 \mathrm{~min}$ (double the time that an experienced user takes the protocol). All participants completed the task within that time, demonstrating the productivity of the task.

In terms of satisfaction, it was reported an average score of 8.3 that reveals high levels of satisfaction with the system. It should be noted that, nevertheless, one of the participants gave 5 points to the level of satisfaction. During the debriefing with this participant, it was possible to realize that this low score was given due to the fact that the participant did not like the GUI of the authoring tool. Although this comment is the only one to mention the design of the GUI, the research team will make efforts to improve not only the design of the GUI but also to optimize the arrangement of the elements that compose it.

\section{Conclusions and Final Remarks}

The evaluation of the multisensory 360 video authoring tool revealed encouraging results as the average usability index obtained was 81.3 , fitting into the Percentile A that corresponds to a pleasant use system and with the potential for recommendation by part of its users. The experimental protocol was developed in such a way that the participants had to use all the functionalities of the tool and, nevertheless, no participant made mistakes in defined tasks. Satisfaction indexes were also high, which shows a good acceptance of the same.

In the debriefing interviews, some participants referred that the design could me "prettier". As future work, it is intended to make a design study to design a more appealing GUI and that optimizes the arrangement of the elements that make up the GUI. In addition, it is intended to implement improvements to existing features as well as implement new features such as 360 3D video support. 
Acknowledgements. This work was also partially supported by the project "DOUROTUR, Turismo e Inovação Tecnológica no Douro/NORTE-01-0145-FEDER000014" financed by the North Portugal Regional Operational Programme (NORTE 2020), under the PORTUGAL 2020 Partnership Agreement, and through the European Regional Development Fund (ERDF). All the works were conducted at INESC TEC's MASSIVE VR Laboratory.

\section{References}

1. Bleumers, L., Van den Broeck, W., Lievens, B., Pierson, J.: Seeing the bigger picture: a user perspective on 360 TV. In: Proceedings of the 10th European Conference on Interactive TV and Video, EuroiTV 2012, pp. 115-124. ACM, New York (2012). https://doi.org/10.1145/2325616.2325640.

2. Brooke, J., et al.: Sus-a quick and dirty usability scale. Usabili. Eval. Ind. 189(194), 4-7 (1996)

3. ButtKicker: The ButtKicker LFE Kit (2017). http://www.thebuttkicker.com/lfekit

4. DiLaura, D.L., Houser, K.W., Mistrick, R.G., Steffy, G.R.: The Lighting Handbook: Reference and Application. Illuminating Engineering Society of North America, New York (2011)

5. Dix, A.: Human-Computer Interaction. Springer, Heidelberg (2009)

6. Freitas, J., Meira, C., Melo, M., Barbosa, L., Bessa, M.: Information system for the management and visualization of multisensorial contents. In: 2015 10th Iberian Conference on Information Systems and Technologies (CISTI), pp. 1-7. IEEE (2015)

7. Fuchs, P., Moreau, G., Guitton, P.: Virtual Reality: Concepts and Technologies. CRC Press, Boca Raton (2011)

8. ISO 9241-125:2017: Ergonomics of human-system interaction. Standard, International Organization for Standardization, Geneva, CH (2017)

9. Israr, A., Schwemler, Z., Mars, J., Krainer, B.: VR360HD: a VR360 player with enhanced haptic feedback. In: Proceedings of the 22nd ACM Conference on Virtual Reality Software and Technology, pp. 183-186. ACM (2016)

10. Kirakowski, J., Corbett, M.: Sumi: the software usability measurement inventory. Br. J. Educ. Technol. 24(3), 210-212 (1993)

11. MarketsandMarkets: Head-Mounted Display Market by Component (Hardware and Software), Application (Aviation \& Tactical, Engineering, Medical, Training $\&$ Simulation, and Others), Product (Head-Mounted and Eyewear), Technology, and Geography - Global Trend and Forecast to 2020. Technical report (2015)

12. Norman, K.L., Shneiderman, B., Harper, B., Slaughter, L.: Questionnaire for user interaction satisfaction. University of Maryland (Norman, 1989) Disponível em (1998)

13. Oculus: Oculus rift — oculus (2017). https://www.oculus.com/rift/

14. Ramalho, J., Chambel, T.: Sensing immersive 360 mobile interactive video. In: 2014 International Conference on Computer Graphics Theory and Applications (GRAPP), pp. 1-10. IEEE (2014)

15. SensoryCo: SmX-4D series - SensoryCo TS (2017). http://www.sensorycots.com/ smx-4d-series

16. Visch, V.T., Tan, E.S., Molenaar, D.: The emotional and cognitive effect of immersion in film viewing. Cogn. Emot. 24(8), 1439-1445 (2010) 